\title{
Portfolio Sensitivity to the Changes in the Maximum and the Maximum Drawdown
}

\author{
Libor Pospisil, Jan Vecer \\ Columbia University, Department of Statistics, New York, NY 10027, USA
}

March 11, 2008

\begin{abstract}
In this article, we define new "Greeks" for financial derivatives: sensitivities to the running maximum and the running maximum drawdown of an underlying asset. Some types of portfolios, such as the net asset value of a hedge fund or performance fees are sensitive to these parameters. In order to illustrate the concept of the new "Greeks", we derive probabilistic representations of sensitivities for two classes of financial contracts: forwards on the maximum drawdown and lookback options. These results allow us to interpret the delta-hedge of the contracts in a novel way.
\end{abstract}

\section{Introduction}

Portfolio sensitivities to various market variables, defined as the derivatives of the portfolio value with respect to these variables, play an important role in risk management. When a portfolio consists of options, the sensitivities are usually denoted by Greek letters and called the "Greeks". The most commonly used Greeks are: delta, vega, theta, and rho - the sensitivities of an option value to the underlying asset price, the volatility of the underlying asset, the time to maturity, and the interest rate, respectively. Another Greek letter, gamma, represents the sensitivity of delta to the underlying asset price. The Greeks, especially delta, gamma, and vega, can be used to hedge a position in an option against the moves of the corresponding market variables.

In this article, we define new types of sensitivities. First, we assume that the value of a financial contract depends not only on the price of an underlying asset, but also on at least one of the following variables: the running maximum of the underlying asset, the running minimum, the running maximum drawdown, or the running maximum drawup. Subsequently, the new sensitivities are defined as the derivatives of the contract value with respect to these variables. We denote these derivatives by Greek letters in order to emphasize the similarity with the other Greeks.

Some types of portfolios are sensitive to the changes of the maximum and the maximum drawdown. Namely performance fees of the hedge fund managers are typically tied to resetting a new "High water mark", representing sensitivity to the maximum net asset value of the fund. Similarly, withdrawal of the investors from a given fund is typically triggered by a maximum drawdown of the net asset value.

In our paper we study forwards on the maximum drawdown or the maximum drawup and lookback options as examples of contracts, whose values depend on some of the previously mentioned variables. We derive the sensitivities of these contracts and show that they have a useful probabilistic representation. In addition, we interpret the deltas of the contracts in a novel way. The main results are presented in Theorems 3.1 and 4.1.

We assume throughout this article that there is a risk-neutral measure and the price of the underlying asset follows a geometric Brownian motion with constant parameters under this measure. However, some results about the sensitivities to the maximum and the maximum drawdown hold under weaker assumptions, which are discussed in Remark 3.2 and 4.2.

A closed form solution to the lookback option pricing problem was derived by Goldman et al. (1979), under 
the assumption that the underlying asset follows a geometric Brownian motion. This result can be used to obtain an analytical formula for the sensitivity of a lookback option, which we define in this article. Hobson (1998) found lower and upper bounds for the price of a lookback option, which are implied by the market values of plain-vanilla call options, and therefore are independent of a model for the underlying asset. Equivalence between floating and fixed strike lookback option was studied in Eberlein and Papapantoleon (2005).

A formula for the expected maximum drawdown of a Brownian motion with a drift, containing integrals of series expansions, is presented in Magdon-Ismail et al. (2004). Pospisil and Vecer (2007) employed numerical methods for partial differential equations to price the maximum drawdown. However, an analytical expression of the expected maximum drawdown has not been derived yet. Therefore, we do not have closed form solutions for the sensitivities of the maximum drawdown.

This article is organized as follows. In Section 2, we define the new Greeks. We calculate the Greeks for a forward on the maximum drawdown in Section 3, and a lookback option in Section 4. Section 5 contains concluding remarks.

\section{Sensitivities to the Maximum and the Maximum Drawdown}

We assume that an asset $S$, which we will call the underlying asset, is traded in a market. Furthermore, let us assume that there exists a risk-neutral probability measure $\mathbb{Q}$, under which process $S$ is a geometric Brownian motion:

$$
d S_{t}=r S_{t} d t+\sigma S_{t} d W_{t}, \quad t \in[0, T] .
$$

$W$ is a Brownian motion under $\mathbb{Q}, r$ is the risk-free interest rate, and $T>0$ is a fixed time. Symbol $\mathcal{F}_{t}$ will denote the $\sigma$-field generated by process $S$ up to time $t: \mathcal{F}_{t}=\sigma\left(S_{u}, u \leq t\right)$.

We define the running maximum $(M)$ and the running minimum $(\underline{M})$ of the underlying asset at time $t$ as the maximum and the minimum value of $S$ for period $[0, \mathrm{t}]$ :

$$
\begin{aligned}
M_{t} & =\max _{u \in[0, t]} S_{u}, \\
\underline{M}_{t} & =\min _{u \in[0, t]} S_{u} .
\end{aligned}
$$

The running maximum drawdown $(M D D)$ at time $t$ is defined as the maximum drop of process $S$ from $M$ on $[0, t]$, while the running maximum drawup $(M D U)$ is defined as the maximum surge of $S$ from $\underline{M}$ :

$$
\begin{aligned}
M D D_{t} & =\max _{u \in[0, t]}\left(M_{u}-S_{u}\right), \\
M D U_{t} & =\max _{u \in[0, t]}\left(S_{u}-\underline{M}_{u}\right) .
\end{aligned}
$$

Let us consider a contract with payoff $V_{T}$ at time $T$, where $V_{T}$ is a function of some of the following variables: $S_{T}, M_{T}, \underline{M}_{T}, M D D_{T}$, or $M D U_{T}$. Since there is a risk-neutral measure $\mathbb{Q}$, we can write the price of this contract at time $t$ as:

$$
V_{t}=e^{-r(T-t)} \mathbb{E}\left[V_{T} \mid \mathcal{F}_{t}\right],
$$

where $\mathbb{E}$ stands for the expected value under $\mathbb{Q}$. Process $S$ defined by equation (1) has the Markov property, therefore $V_{t}$ is a function of $t$ and some of the variables: $S_{t}, M_{t}, \underline{M}_{t}, M D D_{t}$, or $M D U_{t}$. We will denote this function as $v$ and its derivatives with respect to these variables as $v_{s}, v_{m}, v_{\underline{m}}, v_{m d d}$, and $v_{m d u}$. 
We define sensitivities of the contract to the running maximum, the running minimum, the running maximum drawdown, and the running maximum drawup as:

$$
\begin{array}{cl}
\mu=v_{m}, & \underline{\mu}=v_{\underline{m}}, \\
\zeta=v_{m d d}, & \underline{\zeta}=v_{m d u} .
\end{array}
$$

In the following sections, we calculate $\mu, \underline{\mu}, \zeta$, and $\underline{\zeta}$ for forwards on the maximum drawdown, the maximum drawup, and for lookback options.

\section{Forward on the Maximum Drawdown}

A forward on the maximum drawdown of $S$ is a contract with payoff $M D D_{T}$ at time $T$. Its price at time $t$ can be written as:

$$
V_{t}=e^{-r(T-t)} \mathbb{E}\left[M D D_{T} \mid \mathcal{F}_{t}\right] .
$$

The Markov property of process $S$ allows us to express $V_{t}$ as a function of $S_{t}, M_{t}$, and $M D D_{t}$ (see Shreve (2004), pages 107 and 309):

$$
V_{t}=v\left(t, S_{t}, M_{t}, M D D_{t}\right)=e^{-r(T-t)} \mathbb{E}\left[M D D_{T} \mid S_{t}, M_{t}, M D D_{t}\right] .
$$

The sensitivities of the forward to the running maximum and the running maximum drawdown are the corresponding derivatives of function $v(t, s, m, m d d)$ :

$$
\begin{aligned}
\mu_{t} & =\mu\left(t, S_{t}, M_{t}, M D D_{t}\right)=v_{m}\left(t, S_{t}, M_{t}, M D D_{t}\right), \\
\zeta_{t} & =\zeta\left(t, S_{t}, M_{t}, M D D_{t}\right)=v_{m d d}\left(t, S_{t}, M_{t}, M D D_{t}\right) .
\end{aligned}
$$

The delta-hedge of the forward is:

$$
\Delta_{t}=\Delta\left(t, S_{t}, M_{t}, M D D_{t}\right)=v_{s}\left(t, S_{t}, M_{t}, M D D_{t}\right) .
$$

Let us introduce a stopping time $\tau_{M_{t}}$, the first time after $t$ when $S_{u}$ attains the running maximum $M_{t}$. If $S$ does not reach $M_{t}$ on $[t, T]$, we put $\tau_{M_{t}}=T$. Thus,

$$
\tau_{M_{t}}=T \wedge \inf \left\{u \geq t ; S_{u}=M_{t}\right\} .
$$

The maximum drawdown on interval $\left[t, \tau_{M_{t}}\right]$ is:

$$
M D D_{\left[t, \tau_{M_{t}}\right]}=M_{t}-\min _{u \in\left[t, \tau_{M_{t}}\right]}\left(S_{u}\right) .
$$

We set $M D D_{[t, t]}$ to be equal to zero.

In Theorem 3.1, we present probabilistic representations of $\mu_{t}, \zeta_{t}$, and $\Delta_{t}$.

Theorem 3.1 Let $v$ be the value function of a forward on the maximum drawdown, defined in (3), and let $t$ be the actual time, $t \in[0, T]$. Then

$$
\begin{gathered}
v\left(t, S_{t}, M_{t}, M D D_{t}\right) \geq e^{-r(T-t)} M D D_{t}, \\
\zeta_{t}=e^{-r(T-t)} \mathbb{Q}\left[M D D_{T}=M D D_{t} \mid S_{t}, M_{t}, M D D_{t}\right], \\
\mu_{t}=e^{-r(T-t)} \mathbb{Q}\left[M D D_{T}=M D D_{\left[t, \tau_{M_{t}}\right]} \mid S_{t}, M_{t}, M D D_{t}\right], \\
\Delta_{t}=\frac{1}{S_{t}}\left(V_{t}-M_{t} \cdot \mu_{t}-M D D_{t} \cdot \zeta_{t}\right) .
\end{gathered}
$$


Proof:

Proof of inequality (5):

According to the definition of the maximum drawdown, $M D D_{T} \geq M D D_{t}$. Thus,

$$
\begin{aligned}
v\left(t, S_{t}, M_{t}, M D D_{t}\right) & =e^{-r(T-t)} \mathbb{E}\left[M D D_{T} \mid S_{t}, M_{t}, M D D_{t}\right] \\
& \geq e^{-r(T-t)} M D D_{t} .
\end{aligned}
$$

Proof of equation (6):

When $t=T$, formula (6) is true because $v(T, s, m, m d d)=m d d$ and therefore both sides of the formula are one. Hence, we will assume that $t<T$ throughout this proof. Let us introduce the following notation:

$M D D_{[t, T]}=\max _{u \in[t, T]}\left(M_{u}-S_{u}\right)$ and

$$
\begin{array}{ll}
\mathbb{E}_{t}^{m d d}[\cdot]=\mathbb{E}\left[\cdot \mid S_{t}, M_{t}, M D D_{t}=m d d\right], & \mathbb{E}_{t}[\cdot]=\mathbb{E}\left[\cdot \mid S_{t}, M_{t}\right], \\
\mathbb{Q}_{t}^{m d d}[\cdot]=\mathbb{Q}\left[\cdot \mid S_{t}, M_{t}, M D D_{t}=m d d\right], & \mathbb{Q}_{t}[\cdot]=\mathbb{Q}\left[\cdot \mid S_{t}, M_{t}\right] .
\end{array}
$$

Let $F_{M D D_{[t, T]}}(z)$ and $f_{M D D_{[t, T]}}(z)$ be the conditional distribution function and the conditional density function of $M D D_{[t, T]}$. Using the independence of $M D D_{[t, T]}$ and $M D D_{t}$ given $\left(S_{t}, M_{t}\right)$, the expected maximum drawdown is:

$$
\begin{aligned}
v\left(t, S_{t}, M_{t}, m d d\right)= & e^{-r(T-t)} \mathbb{E}_{t}^{m d d}\left[M D D_{T}\right] \\
= & e^{-r(T-t)} \mathbb{E}_{t}^{m d d}\left[M D D_{T} \mathbb{I}_{\left\{M D D_{[t, T]}>M D D_{t}\right\}}\right] \\
& +e^{-r(T-t)} \mathbb{E}_{t}^{m d d}\left[M D D_{T} \mathbb{I}_{\left\{M D D_{[t, T]} \leq M D D_{t}\right\}}\right] \\
= & e^{-r(T-t)} \mathbb{E}_{t}\left[M D D_{[t, T]} \mathbb{I}_{\left\{M D D_{[t, T]}>m d d\right\}}\right]+e^{-r(T-t)} m d d \mathbb{Q}_{t}\left[M D D_{[t, T]} \leq m d d\right] \\
= & e^{-r(T-t)} \int_{m d d}^{\infty} z f_{M D D_{[t, T]}}(z) d z+e^{-r(T-t)} m d d F_{M D D_{[t, T]}}(m d d) .
\end{aligned}
$$

Calculating the derivative of the expected maximum drawdown with respect to $m d d$, we have:

$$
\begin{aligned}
v_{m d d}\left(t, S_{t}, M_{t}, m d d\right)= & -e^{-r(T-t)} m d d f_{M D D_{[t, T]}}(m d d)+e^{-r(T-t)} F_{M D D_{[t, T]}}(m d d) \\
& +e^{-r(T-t)} m d d f_{M D D_{[t, T]}}(m d d) \\
= & e^{-r(T-t)} F_{M D D_{[t, T]}}(m d d)=e^{-r(T-t)} \mathbb{Q}_{t}\left[M D D_{[t, T]} \leq m d d\right] \\
= & e^{-r(T-t)} \mathbb{Q}\left[M D D_{T}=m d d \mid S_{t}, M_{t}, m d d\right] .
\end{aligned}
$$

Thus,

$$
\zeta_{t}=e^{-r(T-t)} \mathbb{Q}\left[M D D_{T}=M D D_{t} \mid S_{t}, M_{t}, M D D_{t}\right]
$$

Proof of equation (7):

When $t=T$, then both sides of formula (7) are zero because $v(T, s, m, m d d)=m d d$ is independent of $m$ and $M D D_{\left[t, \tau_{M_{t}}\right]}=M D D_{[T, T]}=0$.

Suppose $t \in[0, T)$ is a fixed time. Throughout this proof, we will assume that $S_{t}<m$, which implies that 


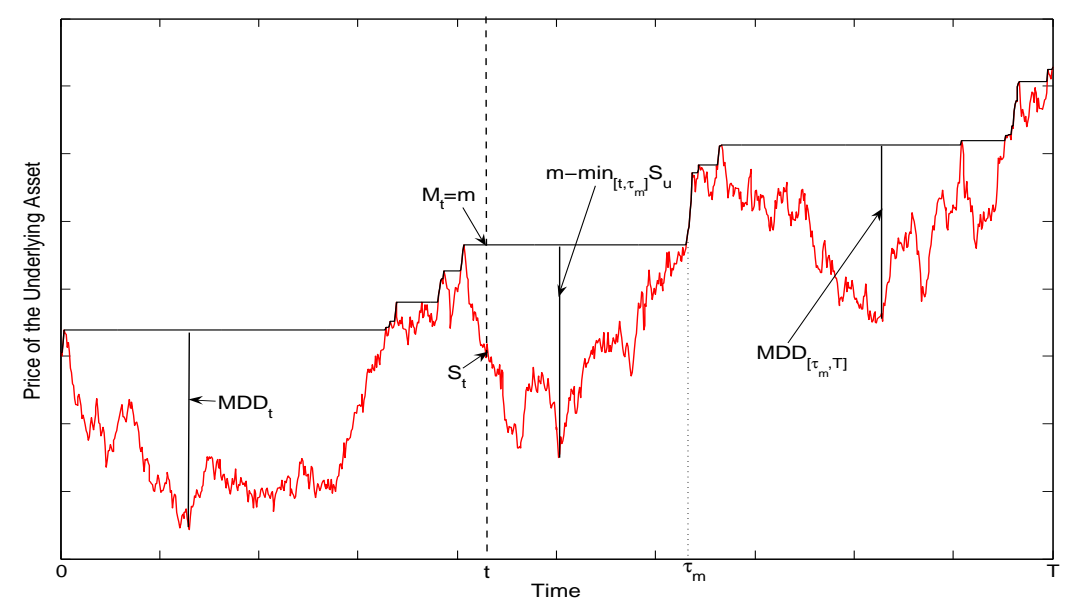

Figure 1: $M D D_{T}$ is attained on one of the three intervals: $[0, t],\left[t, \tau_{m}\right]$, or $\left[\tau_{m}, T\right]$. We use this fact in the proof of formula (7). Notation: $t$ is the actual time; $m$ is the running maximum at $t ; \tau_{m}$ is the first time in the future when $S$ attains $m$ (or $T$ if $S$ does not reach $m$ by the time of maturity).

$\tau_{m}>t$. When $S_{t}=m$ and consequently $\tau_{m}=t$, both sides of (7) are zero and the equation is true (see boundary conditions (9) in Proposition 3.3).

The maximum drawdown must be attained on one of the following time intervals: $[0, t],\left[t, \tau_{m}\right]$, or $\left[\tau_{m}, T\right]$ (see Figure 1). If $M D D_{T}$ is attained on:

- $[0, t]$, then $M D D_{T}=M D D_{t}$.

- $\left[t, \tau_{m}\right]$, then $M D D_{T}=M D D_{\left[t, \tau_{m}\right]}=m-\min _{u \in\left[t, \tau_{m}\right]} S_{u}$. Note that $M_{u}=m$ for $u \in\left[t, \tau_{m}\right]$.

- $\left[\tau_{m}, T\right]$, then $M D D_{T}=M D D_{\left[\tau_{m}, T\right]}=\max _{u \in\left[\tau_{m}, T\right]}\left(M_{u}-S_{u}\right)$. Note that $M_{\tau_{m}}=m$ and $S_{\tau_{m}}=m$ if $\tau_{m}<T$.

Let us introduce a new notation for this part of the proof:

$$
\begin{array}{ll}
\mathbb{E}_{t}^{m}[\cdot]=\mathbb{E}\left[\cdot \mid S_{t}, M_{t}=m, M D D_{t}\right], & \mathbb{E}_{t}[\cdot]=\mathbb{E}\left[\cdot \mid S_{t}, M D D_{t}\right], \\
\mathbb{Q}_{t}^{m}[\cdot]=\mathbb{Q}\left[\cdot \mid S_{t}, M_{t}=m, M D D_{t}\right], & \mathbb{Q}_{t}[\cdot]=\mathbb{Q}\left[\cdot \mid S_{t}, M D D_{t}\right] .
\end{array}
$$

Writing the maximum drawdown as a function of $m$ :

$$
\begin{aligned}
M D D_{T}(m)= & M D D_{t} \mathbb{I}_{\left\{M D D_{T}=M D D_{t}\right\}}+M D D_{\left[t, \tau_{m}\right]} \mathbb{I}_{\left\{M D D_{T}=M D D_{\left[t, \tau_{m}\right]}\right\}} \\
& +M D D_{\left[\tau_{m}, T\right]} \mathbb{I}_{\left\{M D D_{T}=M D D_{\left[\tau_{m}, T\right]}\right\}} \\
= & M D D_{t} \mathbb{I}_{\left\{M D D_{t} \geq \max \left(m-\min _{\left[t, \tau_{m}\right]} S_{u}, M D D_{\left[\tau_{m}, T\right]}\right)\right\}} \\
& +\left(m-\min _{u \in\left[t, \tau_{m}\right]} S_{u}\right) \mathbb{I}_{\left\{m-\min _{\left[t, \tau_{m}\right]} S_{u} \geq \max \left(M D D_{t}, M D D_{\left[\tau_{m}, T\right]}\right)\right\}} \\
& +M D D_{\left[\tau_{m}, T\right]} \mathbb{I}_{\left\{M D D_{\left[\tau_{m}, T\right]} \geq \max \left(M D D_{t}, m-\min _{\left[t, \tau_{m}\right]} S_{u}\right)\right\}} .
\end{aligned}
$$

These equalities hold on set:

$$
A=\left\{\omega \in \Omega ; M D D_{T} \text { is greater than two of the values :MDD }, m-\min _{u \in\left[t, \tau_{m}\right]} S_{u}, M D D_{\left[\tau_{m}, T\right]}\right\} .
$$


This set has probability one, $\mathbb{Q}(A)=1$, because random variables $m-\min _{u \in\left[t, \tau_{m}\right]} S_{u}$ and $M D D_{\left[\tau_{m}, T\right]}$ have continuous distributions on $(0, m)$ and $(0, \infty)$, respectively. $M D D_{\left[\tau_{m}, T\right]}$ has an atom at 0 , but this fact does not affect $\mathbb{Q}(A)$. Since $\left(S_{u}\right)_{u \in[t, T]}$ is independent of $M_{t}$ given $S_{t}$, we have

$$
E_{t}^{m}\left[M D D_{T}\right]=E_{t}\left[M D D_{T}(m)\right]
$$

We can see that $M D D_{T}(m)$ depends on $m$ through three terms: $m, \min _{u \in\left[t, \tau_{m}\right]} S_{u}$, and $M D D_{\left[\tau_{m}, T\right]}$. We want to find a set $B$ on which these functions of $m$ are continuous. Let us define

$$
B=\left\{\omega \in \Omega ; \lim _{h \rightarrow 0} \tau_{m+h}=\tau_{m}\right\}
$$

for a fixed $m>0$. This set has probability one because

$$
\mathbb{Q}(B)=1-\mathbb{Q}\left[\tau_{m}<T \& \exists \varepsilon>0 ; M_{\tau_{m}+\varepsilon}=m\right]=1 .
$$

The last equality follows from the fact that $M_{\tau_{m}+\varepsilon}$ is a random variable with a continuous distribution. Terms $\min _{u \in[t, x]} S_{u}$ and $M D D_{[x, T]}$ are continuous functions of $x$. Therefore, $\min _{u \in\left[t, \tau_{m}\right]} S_{u}$ and $M D D_{\left[\tau_{m}, T\right]}$ are continuous functions of $m$ for $\omega \in B$.

Let us discuss how $M D D_{T}(m)$ changes if we increase or decrease $m$. First, we assume that $h>0$. There are only three cases when $M D D_{T}(m+h)-M D D_{T}(m) \neq 0$.

(a) If $M D D_{T}(m+h)$ is attained on $\left[t, \tau_{m+h}\right]$ and $M D D_{T}(m)$ on $\left[t, \tau_{m}\right]$,

$$
M D D_{T}(m+h)-M D D_{T}(m)=h .
$$

We can write this event as:

$$
C_{1}=\left\{\omega ; m-\min _{\left[t, \tau_{m}\right]} S_{u} \geq M D D_{t}, m-\min _{\left[t, \tau_{m}\right]} S_{u} \geq M D D_{\left[\tau_{m}, T\right]}\right\}
$$

(b) If $M D D_{T}(m+h)$ is attained on $\left[t, \tau_{m+h}\right]$ and $M D D_{T}(m)$ on $[0, t]$, then:

$$
\operatorname{Diff}_{2}=M D D_{T}(m+h)-M D D_{T}(m)=m+h-\min _{\left[t, \tau_{m+h}\right]} S_{u}-M D D_{t} .
$$

This event can be expressed as:

$$
C_{2}=\left\{\omega ; m+h-\min _{\left[t, \tau_{m+h}\right]} S_{u} \geq M D D_{t} \geq m-\min _{\left[t, \tau_{m}\right]} S_{u}, M D D_{t} \geq M D D_{\left[\tau_{m}, T\right]}\right\} .
$$

It can be shown that $\left|\operatorname{Diff}_{2}\right| \leq h$ on $C_{2}$.

(c) If $M D D_{T}(m+h)$ is attained on $\left[t, \tau_{m+h}\right]$ and $M D D_{T}(m)$ on $\left[\tau_{m}, T\right]$,

$$
\operatorname{Diff}_{3}=M D D_{T}(m+h)-M D D_{T}(m)=m+h-\min _{\left[t, \tau_{m}+h\right]} S_{u}-M D D_{\left[\tau_{m}, T\right]} .
$$

This event can be written as:

$$
C_{3}=\left\{\omega ; m+h-\min _{\left[t, \tau_{m+h}\right]} S_{u} \geq M D D_{\left[\tau_{m}, T\right]} \geq m-\min _{\left[t, \tau_{m}\right]} S_{u}, M D D_{\left[\tau_{m}, T\right]} \geq M D D_{t}\right\} .
$$

The following inequality holds on $C_{3}$ : $\left|\operatorname{Diff}_{3}\right| \leq h$. 
Thus, the finite difference of $M D D_{T}(m)$ is:

$$
\frac{1}{h}\left(M D D_{T}(m+h)-M D D_{T}(m)\right)=\mathbb{I}_{C_{1}}+\frac{1}{h} \operatorname{Diff}_{2} \mathbb{I}_{C_{2}}+\frac{1}{h} \operatorname{Diff}_{3} \mathbb{I}_{C_{3}}
$$

Note that $\left|\frac{1}{h} \operatorname{Diff}_{2}\right|$ and $\left|\frac{1}{h} \operatorname{Diff}_{3}\right|$ are bounded by 1 . Since $m, \min _{u \in\left[t, \tau_{m}\right]} S_{u}$, and $M D D_{\left[\tau_{m}, T\right]}$ are continuous functions of $m$ on $B, \mathbb{I}_{C_{2}}$ and $\mathbb{I}_{C_{3}}$ converge to zero on $A \cap B$ as $h$ goes to zero, where $\mathbb{Q}[A \cap B]=1$. Hence,

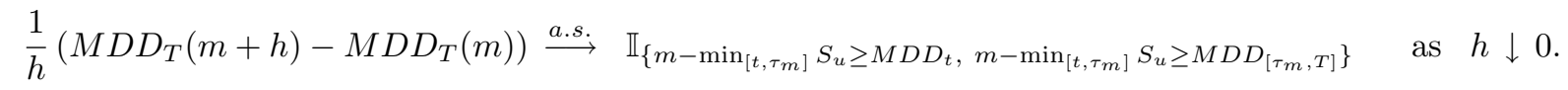

Moreover, this convergence is dominated. If $h<0$, we can use a similar approach to prove that

$$
\frac{1}{h}\left(M D D_{T}(m+h)-M D D_{T}(m)\right) \stackrel{\text { a.s. }}{\longrightarrow} \mathbb{I}_{\left\{m-\min _{\left[t, \tau_{m}\right]} S_{u} \geq M D D_{t}, m-\min _{\left[t, \tau_{m}\right]} S_{u} \geq M D D_{\left[\tau_{m}, T\right]}\right\}} \quad \text { as } h \uparrow 0 .
$$

Term $\frac{1}{h}\left(M D D_{T}(m+h)-M D D_{T}(m)\right)$ is dominated by a sum of three indicator functions, therefore we can conclude that:

$$
\begin{aligned}
v_{m}\left(t, S_{t}, m, M D D_{t}\right) & =\frac{\partial}{\partial m} \mathbb{E}_{t}^{m}\left[M D D_{T}\right] \\
& =\mathbb{E}_{t}\left[\lim _{h \rightarrow 0} \frac{M D D_{T}(m+h)-M D D_{T}(m)}{h}\right] \\
& =\mathbb{Q}_{t}\left[m-\min _{\left[t, \tau_{m}\right]} S_{u} \geq \max \left(M D D_{t}, M D D_{\left[\tau_{m}, T\right]}\right)\right] \\
& =\mathbb{Q}_{t}^{m}\left[M D D_{T}=M D D_{\left[t, \tau_{m}\right]}\right] \\
& =e^{-r(T-t)} \mathbb{Q}\left[M D D_{T}=M D D_{\left[t, \tau_{m}\right]} \mid S_{t}, m, M D D_{t}\right] .
\end{aligned}
$$

Hence,

$$
\mu_{t}=e^{-r(T-t)} \mathbb{Q}\left[M D D_{T}=M D D_{\left[t, \tau_{M_{t}}\right]} \mid S_{t}, M_{t}, M D D_{t}\right] .
$$

Proof of equation (8):

The price of the forward has a linear scaling property:

$$
v(t, \lambda s, \lambda m, \lambda m d d)=\lambda v(t, s, m, m d d), \quad \lambda>0 .
$$

Thus, there is a function $u(t, x, y)$ defined on $[0, T] \times[1, \infty) \times[0, \infty)$, such that

$$
v(t, s, m, m d d)=s \cdot u\left(t, \frac{m}{s}, \frac{m d d}{s}\right) .
$$

Derivatives of $v$ can be expressed in terms of $u$ :

$$
\begin{aligned}
v_{s} & =u-\frac{m}{s} u_{x}-\frac{m d d}{s} u_{y}, \\
v_{m} & =u_{x}, \\
v_{m d d} & =u_{y} .
\end{aligned}
$$

As a result,

$$
\begin{aligned}
\Delta_{t} & =v_{s}\left(t, S_{t}, M_{t}, M D D_{t}\right) \\
& =u-\frac{M_{t}}{S_{t}} \cdot u_{x}-\frac{M D D_{t}}{S_{t}} \cdot u_{y} \\
& =\frac{1}{S_{t}}\left(V_{t}-M_{t} \cdot v_{m}-M D D_{t} \cdot v_{m d d}\right) \\
& =\frac{1}{S_{t}}\left(V_{t}-M_{t} \cdot \mu_{t}-M D D_{t} \cdot \zeta_{t}\right) .
\end{aligned}
$$


Remark 3.2 Formulas (5), (6), and (7) in Theorem 3.1 hold under the following assumptions:

- $S$ is a continuous Markov process under $\mathbb{Q}$.

- $e^{-r t} V_{t}$ is a $\mathbb{Q}$-martingale.

- Random variables $\min _{\left[t, \tau_{m}\right]} S_{u}, M D D_{[t, T]}$, and $M_{\tau_{m}+\varepsilon}$ have continuous distributions under $\mathbb{Q}$, given $\left(S_{t}, M_{t}\right)$, for $t \in[0, T)$ and $\tau_{m}<T$. Stopping time $\tau_{m}$ is defined as $\tau_{m}=T \wedge \inf \left\{u \geq t ; S_{u}=m\right\}$, where $m>S_{t}$ and $\varepsilon$ is a positive number.

Proof: Note that the conditions listed in the Remark were the only assumptions we employed to prove the formulas. In particular, we did not use the fact that $S$ is a geometric Brownian motion. On the other hand, claim (8) does not hold under these weaker assumptions because it is based on the linear scaling property of $v$.

The value function of a forward on the maximum drawdown, $v$, satisfies a partial differential equation, which is presented in Proposition 3.3.

Proposition 3.3 The value function of a forward on the maximum drawdown,

$$
v\left(t, S_{t}, M_{t}, M D D_{t}\right)=e^{-r(T-t)} \mathbb{E}\left[M D D_{T} \mid S_{t}, M_{t}, M D D_{t}\right],
$$

is a solution to the following partial differential equation:

$$
\begin{gathered}
v_{t}+r s v_{s}+\frac{1}{2} \sigma^{2} s^{2} v_{s s}=r v \\
\text { on }(0, T) \times\{(s, m, m d d) ; 0<s<m \& m-s<m d d<m\},
\end{gathered}
$$

with terminal and boundary conditions

$$
\begin{aligned}
v(T, s, m, m d d) & =m d d, & & \\
v(t, s, m, m d d) & =e^{-r(T-t)} m d d & & \text { if } s=0 \text { or } m=m d d, \\
v_{m}(t, s, m, m d d) & =0 & & \text { if } m=s, \\
v_{m d d}(t, s, m, m d d) & =0 & & \text { if } m-s=m d d,
\end{aligned}
$$

where $t \in[0, T)$.

Proof: See Pospisil and Vecer (2007).

Let us discuss the interpretation of Theorem 3.1. Inequality (5) implies that the price of $M D D_{T}$ at time $t$ is at least the discounted value of the actual maximum drawdown $M D D_{t}$.

Formula (6) states that the derivative of the price function $v$ with respect to the actual maximum drawdown $m d d$ is

$$
v_{m d d}\left(t, S_{t}, M_{t}, M D D_{t}\right)=e^{-r(T-t)} \mathbb{Q}\left[M D D_{T}=M D D_{t} \mid S_{t}, M_{t}, M D D_{t}\right] .
$$

The term on the right-hand side can be interpreted as the discounted conditional probability that the maximum drawdown for the entire period $[0, T]$ has been attained before $t$. This probability is zero only if the drawdown at time $t, M_{t}-S_{t}$, equals its running maximum $M D D_{t}$, which is consistent with boundary conditions (9): $v_{m d d}=0$ if $m-s=m d d$. Another interpretation of this result was introduced in the proof of Theorem 3.1: $e^{r(T-t)} v_{m d d}\left(t, S_{t}, M_{t}, m d d\right)$, as a function of $m d d$, is the cumulative distribution function of $M D D_{[t, T]}$ given $S_{t}$ and $M_{t}$.

According to claim (7),

$$
v_{m}\left(t, S_{t}, M_{t}, M D D_{t}\right)=e^{-r(T-t)} \mathbb{Q}\left[M D D_{T}=M D D_{\left[t, \tau_{M_{t}}\right]} \mid S_{t}, M_{t}, M D D_{t}\right] .
$$



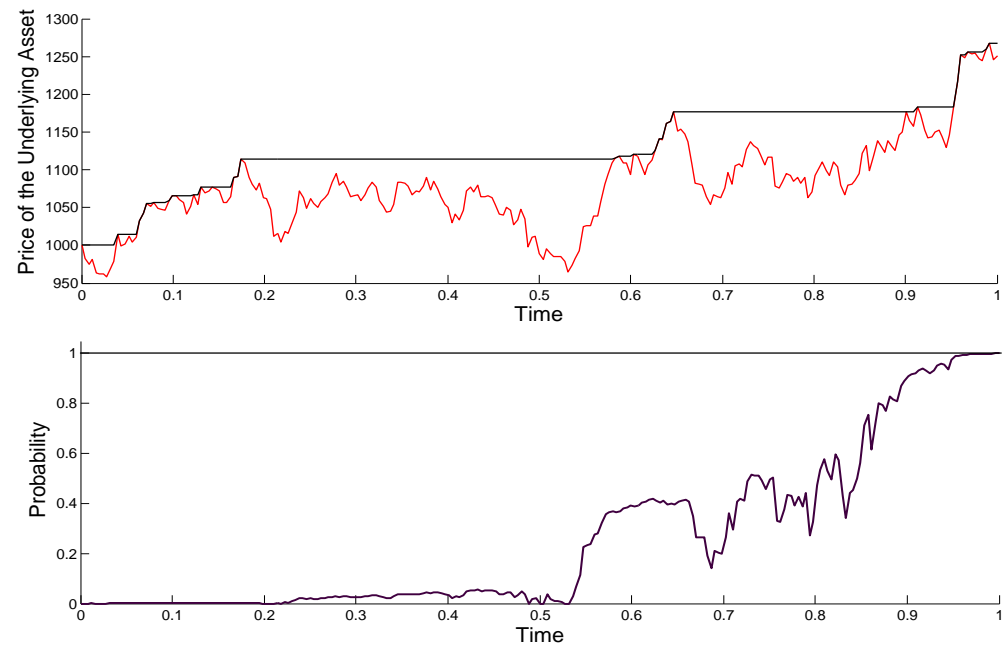

Figure 2: The upper figure contains paths of $S$ and $M$. The corresponding path of $\mathbb{Q}\left[M D D_{T}=M D D_{t} \mid S_{t}, M_{t}, M D D_{t}\right]$, which was calculated as $e^{r(T-t)} v_{m d d}\left(t, S_{t}, M_{t}, M D D_{t}\right)$, is displayed in the lower figure. Process $S$ is a geometric Brownian motion with parameters: $S_{0}=1000, T=1, r=4 \%$, and $\sigma=20 \%$.

Thus, derivative $v_{m}$ can be interpreted as the discounted conditional probability that the maximum drawdown for the entire period $[0, T]$ will be attained between the actual time $t$ and $\tau_{M_{t}}$, the first time after $t$ when $S$ reaches its running maximum $M_{t}$. The probability equals zero only if $S_{t}=M_{t}$, which means that the value of the running maximum is being reset at time $t$ and $\tau_{M_{t}}=t$. This result is consistent with boundary conditions (9): $v_{m}=0$ if $s=m$.

Figures 2-5 illustrate these results. In Figures 2 and 3, we have plotted sample paths of probabilities $\mathbb{Q}\left[M D D_{T}=M D D_{t} \mid S_{t}, M_{t}, M D D_{t}\right]$ and $\mathbb{Q}\left[M D D_{T}=M D D_{\left[t, \tau_{M_{t}}\right]} \mid S_{t}, M_{t}, M D D_{t}\right]$, respectively. Figures 4 and 5 display the probabilities as functions of variables $\left[M_{t}-S_{t}\right] / S_{t}$ and $\left[M D D_{t}-\left(M_{t}-S_{t}\right)\right] / S_{t}$ while keeping the time to maturity fixed at 1 year or 3 months. We can represent the probabilities in this way due to the linear scaling propery of $v$, which we discussed in the proof of Theorem 3.1:

$$
\begin{aligned}
e^{r(T-t)} v_{m d d}(t, s, m, m d d) & =e^{r(T-t)} u_{y}\left(t, \frac{m}{s}, \frac{m d d}{s}\right), \\
e^{r(T-t)} v_{m}(t, s, m, m d d) & =e^{r(T-t)} u_{x}\left(t, \frac{m}{s}, \frac{m d d}{s}\right) .
\end{aligned}
$$

Using formula (8), we can write the price of a forward on the maximum drawdown as:

$$
V_{t}=\Delta_{t} \cdot S_{t}+M_{t} \cdot \mu_{t}+M D D_{t} \cdot \zeta_{t} .
$$

Hence, in order to hedge the forward, an agent must invest amount $B_{t}=M_{t} \cdot \mu_{t}+M D D_{t} \cdot \zeta_{t}$ in the money market. If $B_{t}$ exceeds $V_{t}$, the agent should make up for the difference by shorting the underlying asset. On the other hand, if $B_{t}$ is less than $V_{t}$, the difference $V_{t}-B_{t}$ should be used to purchase the underlying asset. At time $T, V_{T}=M D D_{T}, \zeta_{T}=1$, and $\mu_{T}=0$, which implies $V_{T}=B_{T}$ and $\Delta_{T}=0$. Thus, at the time of maturity the agent should not hold the underlying asset and all the capital should be invested in the money market.

Important properties of the delta-hedge are summarized in Proposition 3.4. 

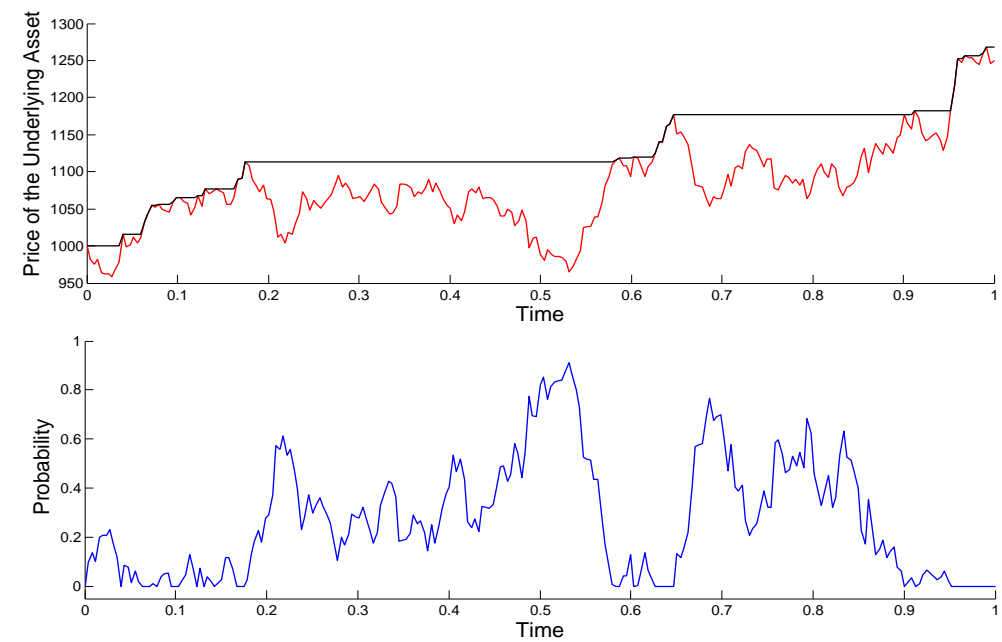

Figure 3: Sample paths of $S$ and its running maximum $M$, plotted in the first graph, are the same as in Figure 2. The path in the second graph represents probability $\mathbb{Q}\left[M D D_{T}=M D D_{\left[t, \tau_{M_{t}}\right]} \mid S_{t}, M_{t}, M D D_{t}\right]$, which was calculated as $e^{r(T-t)} v_{m}\left(t, S_{t}, M_{t}, M D D_{t}\right)$. Process $S$ is a geometric Brwonian motion with parameters: $S_{0}=1000, T=1, r=4 \%$, and $\sigma=20 \%$.

Proposition 3.4 Process $\Delta_{t}$ has the following properties:

(i) $\Delta\left(t, S_{t}, M_{t}, M D D_{t}\right)>-1$ for any $t \in[0, T]$.

(ii) If $S_{t}=M_{t}$, then $\Delta\left(t, S_{t}, M_{t}, M D D_{t}\right) \geq 0$.

(iii) If $S_{0}=M_{0}$ and $M D D_{0}=0$, then $\Delta\left(0, S_{0}, M_{0}, M D D_{0}\right)=\frac{V_{0}}{S_{0}}$.

(iv) $\Delta\left(T, S_{T}, M_{T}, M D D_{T}\right)=0$.

Proof:

(i) + (ii): We will use inequality $V_{t} \geq e^{-r(T-t)} M D D_{t}$ (formula (5)) and the fact that $\zeta_{t} \leq e^{-r(T-t)}$ to prove the first and the second claim:

$$
\begin{aligned}
\Delta_{t}= & \frac{1}{S_{t}}\left(V_{t}-M_{t} \cdot \mu_{t}-M D D_{t} \cdot \zeta_{t}\right) \\
& \geq \frac{1}{S_{t}}\left(V_{t}-e^{-r(T-t)} M D D_{t}-M_{t} \cdot \mu_{t}\right) \\
& \geq-e^{-r(T-t)} \frac{M_{t}}{S_{t}} \mathbb{Q}_{t}\left[M D D_{T}=M D D_{\left[t, \tau_{M_{t}}\right]}\right]
\end{aligned}
$$

By definition, $\frac{M_{t}}{S_{t}} \leq 1$. If $M_{t}=S_{t}$, then $\mathbb{Q}_{t}\left[M D D_{T}=M D D_{\left[t, \tau_{M_{t}}\right]}\right]=0$. This proves inequalities $(i)$ and $(i i)$.

(iii): If $M_{0}=S_{0}$ and $M D D_{0}=0, \mathbb{Q}_{0}\left[M D D_{T}=M D D_{\left[0, \tau_{M_{0}}\right]}\right]=0$, and $\mathbb{Q}_{0}\left[M D D_{T}=M D D_{0}\right]=0$, this implies that $\mu_{0}=0, \zeta_{0}=0$, and thus $\Delta\left(t, S_{t}, M_{t}, M D D_{t}\right)=\frac{V_{0}}{S_{0}}$. 

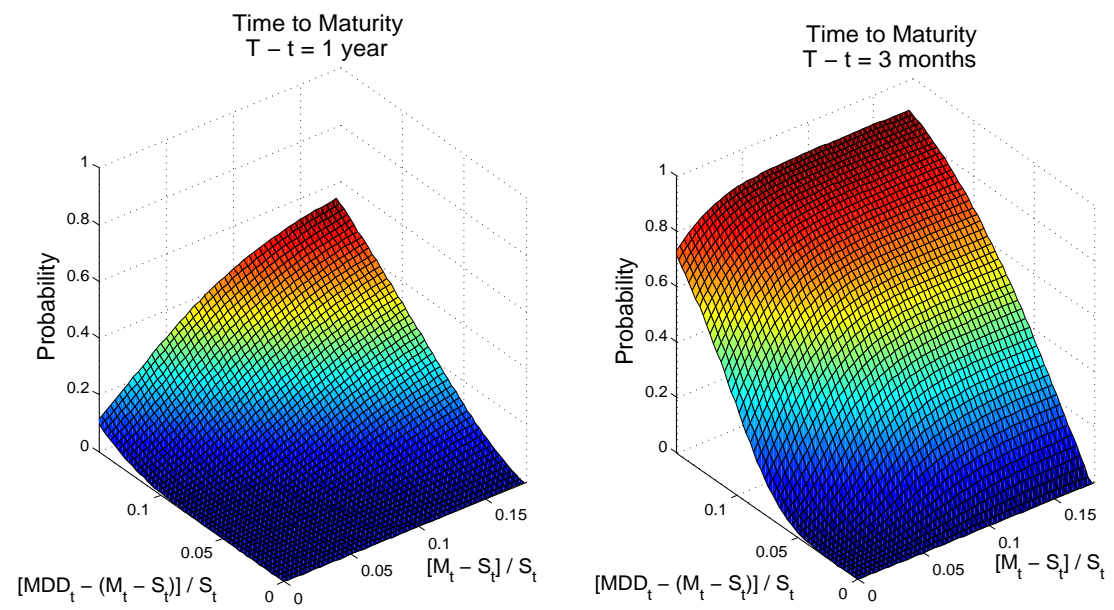

Figure 4: This figure contains plots of probability $\mathbb{Q}\left[M D D_{T}=M D D_{t} \mid S_{t}, M_{t}, M D D_{t}\right]$ for times to maturity 1 year (the left plot) and 3 months (the right plot). The probability is a function of two variables: $\left[M_{t}-S_{t}\right] / S_{t}$ (drop of the asset value $S_{t}$ from its running maximum $M_{t}$, scaled by $S_{t}$ ) and $\left[M D D_{t}-\left(M_{t}-S_{t}\right)\right] / S_{t}$ (distance of the current drawdown $M_{t}-S_{t}$ from the running maximum drawdown $M D D_{t}$, scaled by $S_{t}$ ). Parameters of the model: $r=4 \%$ and $\sigma=20 \%$.

(iv): Since $v(T, s, m, m d d)=m d d$,

$$
\Delta\left(T, S_{T}, M_{T}, M D D_{T}\right)=v_{s}\left(T, S_{T}, M_{T}, M D D_{T}\right)=0 .
$$

Similar results to those we have proved for the maximum drawdown are true for the maximum drawup. We will present them in Theorem 3.5. The value function of a forward on the maximum drawup, $v$, is:

$$
v\left(t, S_{t}, \underline{M}_{t}, M D U_{t}\right)=V_{t}=e^{-r(T-t)} \mathbb{E}\left[M D U_{T} \mid S_{t}, \underline{M}_{t}, M D U_{t}\right] .
$$

Sensitivities of the forward to variables $\underline{m}, m d u$, and $s$ are:

$$
\begin{aligned}
\underline{\mu}_{t} & =v_{\underline{m}}\left(t, S_{t}, \underline{M}_{t}, M D U_{t}\right), \\
\underline{\zeta}_{t} & =v_{m d u}\left(t, S_{t}, \underline{M}_{t}, M D U_{t}\right), \\
\Delta_{t} & =v_{s}\left(t, S_{t}, \underline{M}_{t}, M D U_{t}\right) .
\end{aligned}
$$

We define $\tau_{\underline{M}_{t}}$ as the first time after $t$ when $S_{u}$ drops to the running minimum $\underline{M}_{t}$. If $S$ does not reach $\underline{M}_{t}$ on $[t, T]$, we put $\tau_{\underline{M}_{t}}=T$. Thus,

$$
\tau_{\underline{M}_{t}}=T \wedge \inf \left\{u \geq t ; S_{u}=\underline{M}_{t}\right\} .
$$

The maximum drawup on interval $\left[t, \tau_{\underline{M}_{t}}\right]$ is:

$$
M D U_{\left[t, \tau_{\underline{M}_{t}}\right]}=\max _{u \in\left[t, \underline{\tau}_{\underline{M}}\right]}\left(S_{u}\right)-\underline{M}_{t} .
$$

We set $M D U_{[t, t]}$ to be equal to zero. 

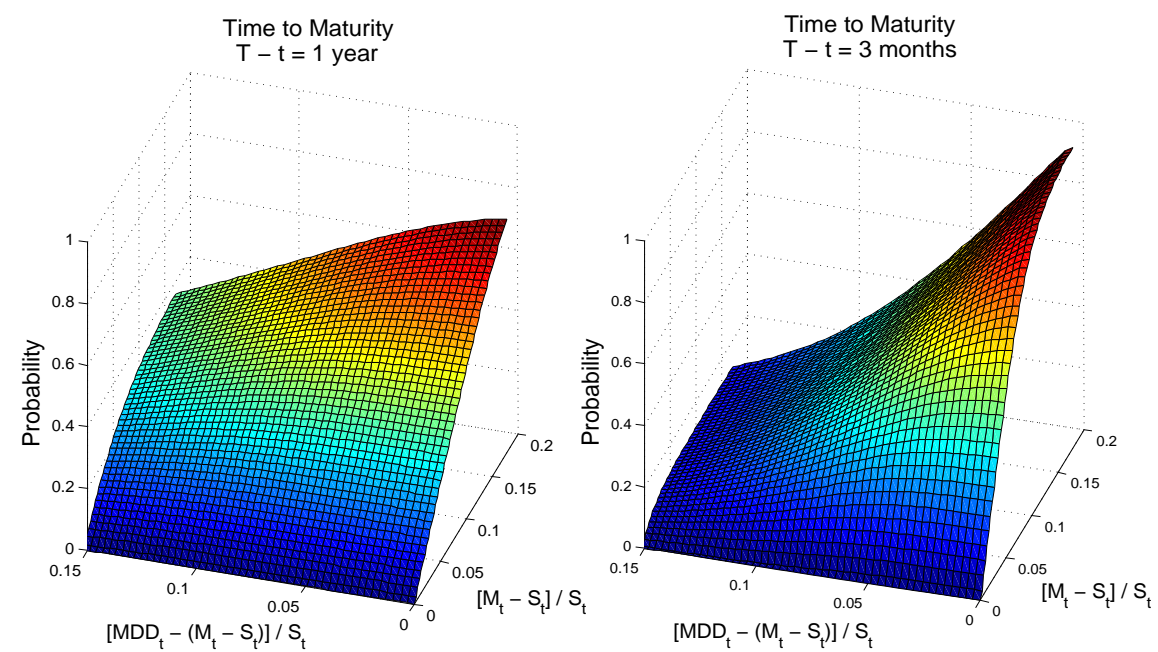

Figure 5: The two plots displayed in this figure represent probability $\mathbb{Q}\left[M D D_{T}=M D D_{\left[t, \tau_{M_{t}}\right]} \mid S_{t}, M_{t}, M D D_{t}\right]$ for two times to maturity: 1 year (the left plot) and 3 months (the right plot). The probability is expressed as a function of two variables: $\left[M_{t}-S_{t}\right] / S_{t}$ (drop of the asset value $S_{t}$ from its running maximum $M_{t}$, scaled by $S_{t}$ ) and $\left[M D D_{t}-\left(M_{t}-S_{t}\right)\right] / S_{t}$ (distance of the current drawdown $M_{t}-S_{t}$ from the running maximum drawdown $M D D_{t}$, scaled by $S_{t}$ ). Parameters of the model: $r=4 \%$ and $\sigma=20 \%$.

Theorem 3.5 Let $v$ be the value function of a forward on the maximum drawup and the actual time, $t \in[0, T]$. Then

$$
\begin{gathered}
v\left(t, S_{t}, \underline{M}_{t}, M D U_{t}\right) \geq e^{-r(T-t)} M D U_{t}, \\
\underline{\zeta}_{t}=e^{-r(T-t)} \mathbb{Q}\left[M D U_{T}=M D U_{t} \mid S_{t}, \underline{M}_{t}, M D U_{t}\right], \\
\left.\underline{\mu}_{t}=-e^{-r(T-t)} \mathbb{Q}\left[M D U_{T}=M D U_{\left[t, \underline{\tau}_{t}\right.}\right] \mid S_{t}, \underline{M}_{t}, M D U_{t}\right], \\
\Delta_{t}=\frac{1}{S_{t}}\left(V_{t}-\underline{M}_{t} \cdot \underline{\mu}_{t}-M D U_{t} \cdot \underline{\zeta}_{t}\right) .
\end{gathered}
$$

PRoOF: Is analogous to the proof of Theorem 3.1.

\section{Lookback Option}

A floating strike lookback call option is a contract with payoff $V_{T}=S_{T}-\underline{M}_{T}$ at the time of maturity. The payoff associated with a floating strike lookback put option is $V_{T}=M_{T}-S_{T}$. Note that a fixed strike lookback put option with a payoff $V_{T}=M_{T}-K$ differs from the floating strike option by $S_{T}-K$, the value of a forward contract which is not sensitive to the changes of the running maximum. Thus we can focus our analysis on the floating strike lookback option without any loss of generality. 
The values of floating strike lookback call and put options are the conditional expectations of the payoffs under the risk-neutral measure $\mathbb{Q}$ :

$$
\begin{aligned}
& \text { Call option: } V_{t}=e^{-r(T-t)} \mathbb{E}\left[S_{T}-\underline{M}_{T} \mid \mathcal{F}_{t}\right], \\
& \text { Put option: } V_{t}=e^{-r(T-t)} \mathbb{E}\left[M_{T}-S_{T} \mid \mathcal{F}_{t}\right]
\end{aligned}
$$

As in the case of a forward on the maximum drawdown, we can use the Markov property of process $S$ to conclude that there exist functions $v(t, s, \underline{m})$ and $v(t, s, m)$, such that:

$$
\begin{aligned}
\text { Call option : } v\left(t, S_{t}, \underline{M}_{t}\right)=V_{t}=e^{-r(T-t)} \mathbb{E}\left[S_{T}-\underline{M}_{T} \mid S_{t}, \underline{M}_{t}\right], \\
\text { Put option : } v\left(t, S_{t}, M_{t}\right)=V_{t}=e^{-r(T-t)} \mathbb{E}\left[M_{T}-S_{T} \mid S_{t}, M_{t}\right] .
\end{aligned}
$$

We define the sensitivities of lookback call and put options to the running minimum and the running maximum, respectively, as the derivatives of functions $v(t, s, \underline{m})$ and $v(t, s, m)$ :

$$
\begin{array}{ll}
\text { Call option : } & \underline{\mu}_{t}=\underline{\mu}\left(t, S_{t}, \underline{M}_{t}\right)=v_{\underline{m}}\left(t, S_{t}, \underline{M}_{t}\right), \\
\text { Put option: } & \mu_{t}=\mu\left(t, S_{t}, M_{t}\right)=v_{m}\left(t, S_{t}, M_{t}\right) .
\end{array}
$$

In the following theorem, we present a probabilistic interpretation of the floating strike lookback put option sensitivity to the running maximum. Symbol $\Delta_{t}$ will denote the delta-hedge of the option:

$$
\Delta_{t}=\Delta\left(t, S_{t}, M_{t}\right)=v_{s}\left(t, S_{t}, M_{t}\right) .
$$

Theorem 4.1 Let $v$ be the value function of a floating strike lookback put option and the current time, $t \in[0, T]$. Then:

$$
\begin{gathered}
\mu_{t}=e^{-r(T-t)} \mathbb{Q}\left[M_{T}=M_{t} \mid S_{t}, M_{t}\right], \quad t \in[0, T] . \\
\Delta_{t}=\frac{1}{S_{t}}\left(V_{t}-M_{t} \cdot \mu_{t}\right), \quad t \in[0, T] .
\end{gathered}
$$

\section{ProOF:}

Proof of equation (10): If $t=T$, both sides of (10) are 1 and the formula is true. Thus, we will assume that $t<T$ in the remaining part of the proof. We will use the following notation for conditional expected values and risk-neutral probabilities:

$$
\begin{array}{ll}
\mathbb{E}_{t}^{m}[\cdot]=\mathbb{E}\left[\cdot \mid S_{t}, M_{t}=m\right], & \mathbb{E}_{t}[\cdot]=\mathbb{E}\left[\cdot \mid S_{t}\right], \\
\mathbb{Q}_{t}^{m}[\cdot]=\mathbb{Q}\left[\cdot \mid S_{t}, M_{t}=m\right], & \mathbb{Q}_{t}[\cdot]=\mathbb{Q}\left[\cdot \mid S_{t}\right] .
\end{array}
$$

The value function can be represented as:

$$
\begin{aligned}
v\left(t, S_{t}, m\right)= & e^{-r(T-t)} \mathbb{E}_{t}^{m}\left[M_{T}-S_{T}\right] \\
= & e^{-r(T-t)} \mathbb{E}_{t}^{m}\left[M_{T}\right]-e^{-r(T-t)} \mathbb{E}_{t}^{m}\left[S_{T}\right] \\
= & e^{-r(T-t)} \mathbb{E}_{t}^{m}\left[M_{T} \mathbb{I}_{\left.\left\{M_{t}<\max _{u \in[t, T]} S_{u}\right\}\right]}\right. \\
& +e^{-r(T-t)} \mathbb{E}_{t}^{m}\left[M_{T} \mathbb{I}_{\left.\left\{M_{t}>\max _{u \in[t, T]} S_{u}\right\}\right]-S_{t}}\right. \\
= & e^{-r(T-t)} \mathbb{E}_{t}\left[\left(\max _{u \in[t, T]} S_{u}\right) \mathbb{I}_{\left.\left\{m \leq \max _{u \in[t, T]} S_{u}\right\}\right]}\right] \\
& +e^{-r(T-t)} m \mathbb{Q}_{t}\left[\max _{u \in[t, T]} S_{u} \leq m\right]-S_{t} \\
= & e^{-r(T-t)} \int_{m}^{\infty} x f_{\max S}(x) d x+e^{-r(T-t)} m F_{\max S}(m)-S_{t} .
\end{aligned}
$$


$F_{\max S}(x)$ and $f_{\max S}(x)$ are the conditional cumulative distribution function and the conditional probability density function of $\max _{u \in[t, T]} S_{u}$, given $S_{t}$. Note that if we condition on $S_{t}$, random variables $M_{t}$ and $\max _{u \in[t, T]} S_{u}$ are independent. We will calculate the derivative of $v$ with respect to $m$ :

$$
\begin{aligned}
v_{m}\left(t, S_{t}, m\right)= & -e^{-r(T-t)} m f_{\max S}(m) \\
& +e^{-r(T-t)} F_{\max S}(m)+e^{-r(T-t)} m f_{\max S}(m) \\
= & e^{-r(T-t)} F_{\max S}(m) \\
= & e^{-r(T-t)} \mathbb{Q}_{t}\left[\max _{u \in[t, T]} S_{u} \leq m\right] \\
= & e^{-r(T-t)} \mathbb{Q}\left[\max _{u \in[t, T]} S_{u} \leq M_{t} \mid S_{t}, M_{t}=m\right] \\
= & e^{-r(T-t)} \mathbb{Q}\left[M_{T}=M_{t} \mid S_{t}, M_{t}=m\right] .
\end{aligned}
$$

Hence,

$$
\mu_{t}=v_{m}\left(t, S_{t}, M_{t}\right)=e^{-r(T-t)} \mathbb{Q}\left[M_{T}=M_{t} \mid S_{t}, M_{t}\right] .
$$

Proof of equation (11): Let us define a function $u$ (see Shreve (2004), pages 312-313):

$$
v(t, s, m)=s \cdot u\left(t, \frac{m}{s}\right) .
$$

The derivatives of $v(t, s, m)$ in terms of $u(t, z)$ are:

$$
\begin{aligned}
v_{s}(t, s, m) & =u\left(t, \frac{m}{s}\right)-\frac{m}{s} u_{z}\left(t, \frac{m}{s}\right), \\
v_{m}(t, s, m) & =u_{z}\left(t, \frac{m}{s}\right) .
\end{aligned}
$$

Now we calculate the delta-hedge:

$$
\begin{aligned}
\Delta_{t} & =v_{s}\left(t, S_{t}, M_{t}\right) \\
& =u\left(t, \frac{M_{t}}{S_{t}}\right)-\frac{M_{t}}{S_{t}} \cdot u_{z}\left(t, \frac{M_{t}}{S_{t}}\right) \\
& =\frac{v\left(t, S_{t}, M_{t}\right)}{S_{t}}-\frac{M_{t}}{S_{t}} \cdot v_{m}\left(t, S_{t}, M_{t}\right) \\
& =\frac{V_{t}}{S_{t}}-\frac{M_{t}}{S_{t}} \cdot \mu_{t} \\
& =\frac{1}{S_{t}}\left(V_{t}-M_{t} \cdot \mu_{t}\right) .
\end{aligned}
$$

Remark 4.2 Formula (10) in Theorem 4.1 holds under the following assumptions:

- $S$ is a continuous Markov process under $\mathbb{Q}$.

- $e^{-r t} V_{t}$ is a $\mathbb{Q}$-martingale.

- Random variable $\max _{u \in[t, T]} S_{u}$ has a continuous distribution under $\mathbb{Q}$, given $S_{t}$, for $t \in[0, T)$.

Proof: The conditions listed in this Remark are the only assumptions we needed to prove formula (10). In particular, we did not use the fact that $S$ is a geometric Brownian motion. Claim (11) is based on the linear scaling property of $v$, therefore it would not hold under these weaker assumptions. 
Remark 4.3 The value function of a floating strike lookback put option admits a closed form solution (see Goldman et al. (1979)):

$$
\begin{aligned}
v(t, s, m)= & m \cdot e^{-r \tau} \cdot N\left(\frac{a-\nu \tau}{\sigma \sqrt{\tau}}\right)-\frac{\sigma^{2}}{2 r} \cdot e^{-r \tau} \cdot s \cdot\left(\frac{m}{s}\right)^{\frac{2 r}{\sigma^{2}}} \cdot N\left(\frac{-a-\nu \tau}{\sigma \sqrt{\tau}}\right) \\
& +\frac{\sigma^{2}}{2 r} \cdot s-\left(1+\frac{\sigma^{2}}{2 r}\right) \cdot s \cdot N\left(\frac{a-\left(\nu+\sigma^{2}\right) \tau}{\sigma \sqrt{\tau}}\right)
\end{aligned}
$$

implying

$$
\begin{aligned}
\Delta=v_{s}(t, s, m)= & \frac{\sigma^{2}}{2 r}-\left(1+\frac{\sigma^{2}}{2 r}\right) \cdot N\left(\frac{a-\left(\nu+\sigma^{2}\right) \tau}{\sigma \sqrt{\tau}}\right) \\
& +\left(1-\frac{\sigma^{2}}{2 r}\right) \cdot e^{-r \tau} \cdot\left(\frac{m}{s}\right)^{\frac{2 r}{\sigma^{2}}} \cdot N\left(\frac{-a-\nu \tau}{\sigma \sqrt{\tau}}\right), \\
\mu=v_{m}(t, s, m)= & e^{-r \tau} \cdot N\left(\frac{a-\nu \tau}{\sigma \sqrt{\tau}}\right)-e^{-r \tau} \cdot\left(\frac{m}{s}\right)^{\left(\frac{2 r}{\sigma^{2}}-1\right)} \cdot N\left(\frac{-a-\nu \tau}{\sigma \sqrt{\tau}}\right),
\end{aligned}
$$

where $a=\log \left(\frac{m}{s}\right), \nu=r-\frac{\sigma^{2}}{2}$, and $\tau=T-t$. One can easily check that

$$
v(t, s, m)=s \cdot v_{s}(t, s, m)+m \cdot v_{m}(t, s, m),
$$

which is equivalent to formula (11) in Theorem 4.1.
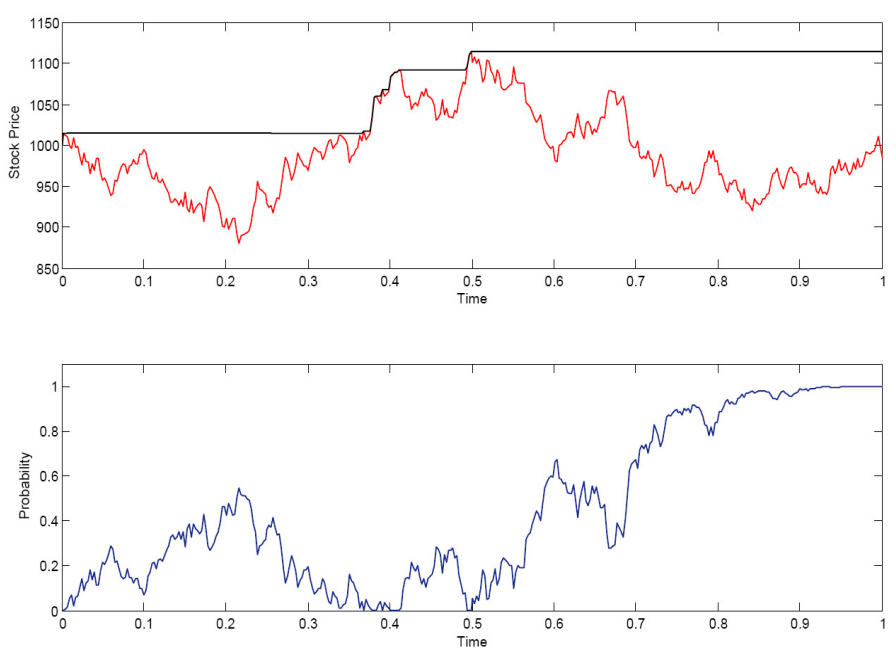

Figure 6: The upper figure contains paths of $S$ and $M$. The lower figure shows the corresponding path of $\mathbb{Q}\left[M_{T}=M_{t} \mid S_{t}, M_{t}\right]$, which was calculated as $e^{r(T-t)} v_{m}\left(t, S_{t}, M_{t}\right)$. Process $S$ is a geometric Brownian motion with parameters: $S_{0}=1000, T=1, r=4 \%$, and $\sigma=20 \%$.

The value function of a floating strike lookback put option, $v$, solves the partial differential equation which is presented in the following proposition.

Proposition 4.4 The value function of a floating strike lookback put option,

$$
v\left(t, S_{t}, M_{t}\right)=e^{-r(T-t)} \mathbb{E}\left[M_{T}-S_{T} \mid S_{t}, M_{t}\right], \quad t \in[0, T],
$$




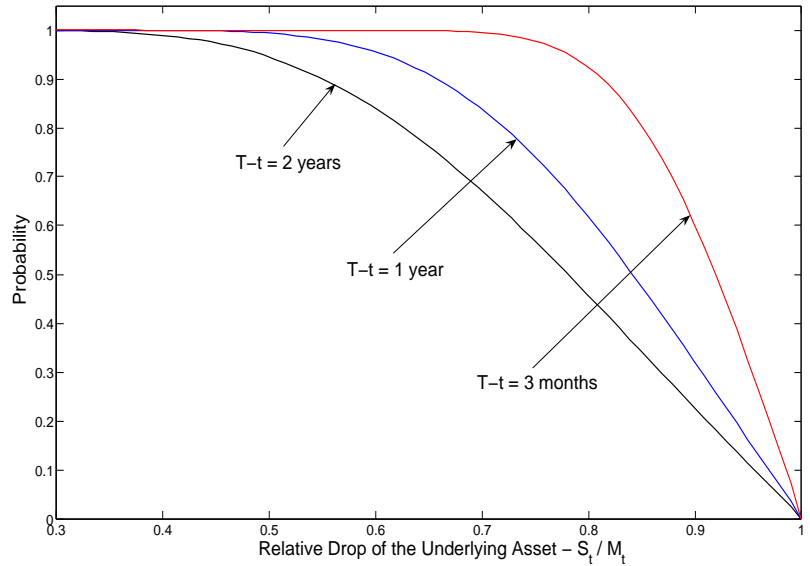

Figure 7: This figure displays probability $\mathbb{Q}\left[M_{T}=M_{t} \mid S_{t}, M_{t}\right]$ as a function of $\frac{S_{t}}{M_{t}}$, which we call the relative drop. We calculated the probability for three different times to maturity: 3 months, 1 year, and 2 years. Process $S$ is geometric Brownian motion with parameters: $r=4 \%$ and $\sigma=20 \%$.

satisfies the following partial differential equation:

$$
\begin{gathered}
v_{t}+r s v_{s}+\frac{1}{2} \sigma^{2} s^{2} v_{s s}=r v \\
\text { on }(0, T) \times\{(s, m) ; 0<s<m\},
\end{gathered}
$$

with terminal and boundary conditions:

$$
\begin{array}{rll}
v(T, s, m) & =m-s, & \\
v(t, s, m) & =e^{-r(T-t)} m & \text { if } s=0 \text { or } m=m d d \\
v_{m}(t, s, m) & =0 & \text { if } m=s,
\end{array}
$$

where $t \in[0, T]$.

Proof: See Shreve (2004), page 309.

Let us discuss the results presented in Theorem 4.1. Equality (10),

$$
v_{m}\left(t, S_{t}, M_{t}\right)=e^{-r(T-t)} \mathbb{Q}\left[M_{T}=M_{t} \mid S_{t}, M_{t}\right],
$$

implies that the derivative of $v$ with respect to the running maximum can be interpreted as the discounted conditional probability that the price process $S$ will not attain the running maximum on interval $[t, T]$. This probability equals zero if $S_{t}=M_{t}$, which is consistent with boundary conditions (12): $v_{m}(t, s, m)=0$ if $s=m$. In the proof of Theorem 4.1, we showed that $e^{r(T-t)} v_{m}\left(t, S_{t}, m\right)$, as a function of $m$, is also the cumulative distribution function of $\max _{[t, T]} S_{u}$ given $S_{t}$.

Figure 6 contains a path of $S$ and the corresponding probability $\mathbb{Q}\left[M_{T}=M_{t} \mid S_{t}, M_{t}\right]$. In Figure 7 , we have plotted this probability as a function of $\frac{s}{m}$ for three times to maturity: 3 months, 1 year, and 2 years. Note that in the proof of Theorem 4.1, we used the following equality:

$$
v_{m}(t, s, m)=u_{z}\left(t, \frac{m}{s}\right) .
$$


Therefore, the probability depends on variables $s$ and $m$ only through ratio $\frac{s}{m}$.

According to Theorem 4.1, we can write the value of a lookback put option as follows:

$$
V_{t}=\Delta_{t} \cdot S_{t}+M_{t} \cdot e^{-r(T-t)} \mathbb{Q}\left[M_{T}=M_{t} \mid S_{t}, M_{t}\right] .
$$

This representation allows us to interpret the dynamic hedging strategy for the option in a novel way. At each time $t \in[0, T]$, an agent should invest the discounted value of $M_{t}$, multiplied by the probability that the maximum for the entire period $[0, T]$ will be $M_{t}$, in the money market. If this amount is lower than $V_{t}$, the difference should be invested in the underlying asset. If the amount invested in the money market exceeds $V_{t}$, the agent should short the underlying asset to make up for the difference.

Let us consider two cases: $S_{t}=M_{t}$, and $S_{t}$ being small compared to $M_{t}$. If the running maximum is being reset, that is when $S_{t}=M_{t}$, then $\mathbb{Q}\left[M_{T}=M_{t} \mid S_{t}, M_{t}\right]=0$ and the entire amount $V_{t}$ should be invested in the underlying asset in order to hedge the option. On the other hand, when $S_{t}$ is small compared to $M_{t}$, then $\mathbb{Q}\left[M_{T}=M_{t} \mid S_{t}, M_{t}\right]$ will be close to one and $V_{t}$ close to $e^{-r(T-t)} M_{t}-S_{t}$. Hence, $\Delta_{t}$ will be approximately negative one, which means that the agent should short one share of the stock and invest $e^{-r(T-t)} M_{t}$ in the money market.

Sensitivities of a lookback call option, $\underline{\mu}_{t}$ and $\Delta_{t}$, have similar probabilistic representations as those of a lookback put option. We present this result in Theorem 4.5.

Theorem 4.5 Let $v$ be the value function of a floating strike lookback call option and $t$ actual time, $t \in[0, T]$. Then:

$$
\begin{gathered}
\underline{\mu}_{t}=-e^{-r(T-t)} \mathbb{Q}\left[\underline{M}_{T}=\underline{M}_{t} \mid S_{t}, \underline{M}_{t}\right], \\
\Delta_{t}=\frac{1}{S_{t}}\left(V_{t}-\underline{M}_{t} \cdot \underline{\mu}_{t}\right) .
\end{gathered}
$$

Proof: Is analogous to the proof of Theorem 4.1.

Finally, let us point out that Theorems 4.1 and 4.5 can be viewed as a generalization of similar formulas for plain vanilla options. If $v\left(t, S_{t}, K\right)=e^{-r(T-t)} \mathbb{E}\left[\left(K-S_{T}\right)^{+} \mid S_{t}\right]$ is the value function of a European put option, the Black-Scholes formula can be used to show that

$$
\begin{aligned}
v_{K}\left(t, S_{t}, K\right) & =e^{-r(T-t)} \mathbb{Q}\left[S_{T} \leq K \mid S_{t}\right], \\
v\left(t, S_{t}, K\right) & =\Delta_{t} \cdot S_{t}+e^{-r(T-t)} K \cdot \mathbb{Q}\left[S_{T} \leq K \mid S_{t}\right] .
\end{aligned}
$$

We can see that formulas (10) and (11) are analogies to these properties of the plain-vanilla option.

\section{Conclusion}

In this article, we introduced new "Greeks" - sensitivities of a portfolio value to the running maximum $(\mu)$, the running minimum $(\underline{\mu})$, the running maximum drawdown $(\zeta)$, and the running maximum drawup $(\underline{\zeta})$.

We calculated these sensitivities for a forward on the maximum drawdown, the maximum drawup, and for a lookback option, and derived probabilistic representations of the sensitivities and the delta-hedge. These results can be used to monitor risk-neutral probabilities that an asset price will not reach its running maximum by the time of maturity, or that the maximum drawdown for a period $[0, T]$ has been attained on $[0, t]$, where $t<T$. 


\section{References}

[1] Eberlein, E., A. Papapantoleon (2005): Equivalence of floating and fixed strike Asian and lookback options, Stochastic Processes and their Applications, Vol. 115, No. 1, 31-40.

[2] Goldman, M. B., H. B. Sosin, M. A. Gatto (1979): Path Dependent Options: "Buy at the Low, Sell at the High", The Journal of Finance, Vol. 34, No. 5, 1111-1127.

[3] Hobson, D. G. (1998): Robust Hedging of the Lookback Option, Finance and Stochastics, Vol. 2, No. $4,329-347$.

[4] Magdon-Ismail, M., A. Atiya, A. Pratap, and Y. Abu-Mostafa (2004): On the Maximum Drawdown of a Brownian Motion, Journal of Applied Probability, Vol. 41, No. 1.

[5] Pospisil, L., J. Vecer (2007): PDE Methods for the Maximum Drawdown, Preprint.

[6] Shreve, S. (2004): Stochastic Calculus for Finance II, Springer Verlag. 